United Nations Educational Scientific and Cultural Organization

and

International Atomic Energy Agency

THE ABDUS SALAM INTERNATIONAL CENTRE FOR THEORETICAL PHYSICS

\title{
ERROR ESTIMATES FOR DISCRETIZED QUANTUM STOCHASTIC DIFFERENTIAL INCLUSIONS
}

\author{
E.O. Ayoola \\ Department of Mathematics, University of Ibadan, \\ Ibadan, Federal Republic of Nigeria ${ }^{1}$ \\ and
}

The Abdus Salam International Centre for Theoretical Physics, Trieste, Italy.

\begin{abstract}
This paper is concerned with the error estimates involved in the solution of a discrete approximation of a quantum stochastic differential inclusion (QSDI). Our main results rely on certain properties of the averaged modulus of continuity for multivalued sesquilinear forms associated with QSDI. We obtained results concerning the estimates of the Hausdorff distance between the set of solutions of the QSDI and the set of solutions of its discrete approximation. This extends the results of Dontchev and Farkhi [6] concerning classical differential inclusions to the present noncommutative quantum setting involving inclusions in certain locally convex space.
\end{abstract}

MIRAMARE - TRIESTE

September 2001

\footnotetext{
${ }^{1}$ Permanent address. E-mail: uimath @ mail.skannet.com
} 


\section{INTRODUCTION}

In continuation of our work in [3, 4] concerning numerical procedures for QSDI, this paper is concerned with the development, analysis and error estimates involved in a one - step discrete scheme for solving quantum stochastic differential inclusion :

$$
\begin{aligned}
d X(t) & \in E(t, X(t)) d \wedge_{\pi}(t)+F(t, X(t)) d A_{f}(t)+G(t, X(t)) d A_{g}^{+}(t) \\
& +H(t, X(t)) d t, \quad \text { almost all } \quad t \in[0, T] \\
X(0) & =X^{0}
\end{aligned}
$$

The coefficients $E, F, G, H$ in (1.1) are elements of $L_{l o c}^{2}([0, T] \times \tilde{\mathcal{A}})_{m v s}$, and the integrators $\wedge_{\pi}, A_{f}, A_{g}^{+}:[0, T] \rightarrow \tilde{\mathcal{A}}$ are the gauge, annihilation and creation processes. The locally convex space $\tilde{\mathcal{A}}$ is the completion of the linear space $L^{+}\left(\mathbb{D} \underline{\otimes} \mathbb{E}, \mathcal{R} \otimes \Gamma\left(L_{\gamma}^{2}\left(\mathbb{R}_{+}\right)\right)\right)$in the Hausdorff topology generated by the family of seminorms $\left\{\|x\|_{\eta \xi}=\mid\langle\eta, x \xi>|: x \in \tilde{\mathcal{A}}, \eta, \xi \in \mathbb{D} \underline{\otimes} \mathbb{E}\right\}$. A solution $X(t)$ of (1.1) is a densely defined linear operator lying in $\operatorname{Ad}(\tilde{\mathcal{A}})_{\text {wac }}$ (see $[3,4,8,9$, 10] for details).

For arbitrary $\eta, \xi \in \mathbb{D} \underline{\otimes} \mathbb{E}$, the equivalent form of (1.1) is the first order initial value nonclassical inclusion given by

$$
\begin{gathered}
\frac{d}{d t}<\eta, X(t) \xi>\in P(t, X(t))(\eta, \xi) \\
X(0)=X^{0}, \text { almost all } t \in[0, T],
\end{gathered}
$$

where $(\eta, \xi) \rightarrow P(t, X(t))(\eta, \xi)$ is a multivalued sesquilinear form on $\mathbb{D} \underline{\otimes} \mathbb{E}$ with values in the field of complex numbers. The explicit form of the map $P(t, x)(\eta, \xi)$ is described as follows:

For $(t, x) \in \mathbb{R}_{+} \times \tilde{\mathcal{A}}, \quad \eta, \xi \in \mathbb{D} \underline{\otimes} \mathbb{E}$ such that $\eta=c \otimes e(\alpha), \quad \xi=d \otimes e(\beta), c, d \in \mathbb{D}$, $\alpha, \beta \in L_{\gamma, l o c}^{\infty}\left(\mathbb{R}_{+}\right)$, define

$$
P_{\alpha \beta}:[0, T] \times \tilde{\mathcal{A}} \rightarrow 2^{\tilde{\mathcal{A}}}
$$

by

$$
P_{\alpha \beta}(t, x)=\mu_{\alpha \beta}(t) E(t, x)+\nu_{\beta}(t) F(t, x)+\sigma_{\alpha}(t) G(t, x)+H(t, x)
$$

where

$$
\mu_{\alpha \beta}(t)=<\alpha(t), \pi(t) \beta(t)>_{\gamma}, \nu_{\beta}(t)=<f(t), \beta(t)>_{\gamma}, \sigma_{\alpha}(t)=<\alpha(t), g(t)>_{\gamma} .
$$

This leads to the multifunction $P:[0, T] \times \tilde{\mathcal{A}} \rightarrow 2^{\operatorname{ses} q(\mathbb{D} \underline{\otimes} \mathbb{E})}$ defined by

$$
P(t, x)(\eta, \xi):=<\eta, P_{\alpha \beta}(t, x) \xi>=\left\{<\eta, Z(t, x) \xi>: Z(t, x) \in P_{\alpha \beta}(t, x)\right\} .
$$


In what follows, we employ the basic function spaces, the set theoretic operations and the Hausdorff metric as in [3, 4, 8 - 10].

The plan for the rest of the paper is as follows: Section 2 contains preliminary statements and basic results in respect of the modulus of continuity of multivalued sesquilinear forms associated with QSDI (1.1). The main result of this paper concerning the error estimates of the discretized inclusion is established in Section 3. This extends and compliments the results of reference [6] concerning similar discretizations of classical differential inclusions.

\section{PRELIMINARY STATEMENTS AND ESTIMATES}

We present in this section, some notations, definitions and estimates which we will employ in the sequel. Without loss of generality, we consider inclusion (1.1) and (1.2) defined only on the interval $[0,1] \subseteq \mathbb{R}_{+}$. Any other interval $[0, T]$, for $T>0$ may be converted into $[0,1]$ by an appropriate regular transformation. In what follows, unless otherwise indicated, $\eta, \xi \in \mathbb{D} \underline{\otimes} \mathbb{E}$ such that $\eta=c \otimes e(\alpha), \xi=d \otimes e(\beta), c, d \in \mathbb{D}, \alpha, \beta \in L_{\gamma, l o c}^{\infty}\left(\mathbb{R}_{+}\right)$.

Let $P:[0,1] \times \tilde{\mathcal{A}} \rightarrow 2^{\text {sesq }(\mathbb{D} \underline{\otimes} \mathbb{E})}$ be a multivalued sesquilinear form satisfying the following conditions:

$\mathcal{S}_{(i)}$ For every $x \in \tilde{\mathcal{A}}, t \in[0,1], P(t, x)(\eta, \xi)$ is nonempty, compact and and convex subset of $\mathscr{C}$ the complex field.

$\mathcal{S}_{(i i)}$ The map $P(t, \cdot)(\eta, \xi)$ is locally Lipschitzian uniformly in $t \in[0,1]$, with Lipschitz constants $K_{\eta, \xi}$.

$\mathcal{S}_{(i i i)}$ There exists positive constants $L$ and $A$ such that

$$
|P(t, x)(\eta, \xi)| \leq L\|x\|_{\eta \xi}+A
$$

for all $x \in \tilde{\mathcal{A}}, t \in[0,1]$ and for all $\eta, \xi \in \mathbb{D} \underline{\otimes} \mathbb{E}$.

Here,

$$
|P(t, x)(\eta, \xi)|=\sup _{Z_{\eta \xi} \in P(t, x)(\eta, \xi)}\left|Z_{\eta \xi}\right| .
$$

We note that the map $t \rightarrow P(t, x)(\eta, \xi)$ is measurable in $[0,1]$ for every fixed element $x \in \tilde{\mathcal{A}}$ (see [8]) since the map is locally integrable on $\mathbb{R}_{+}$.

In our subsequent analysis, we shall need the following Theorem which is the basic existence result of (1.1) due to reference [8]. 
Theorem 2.1. Let $\theta$ be a positive number and $I=\left[t_{0}, T\right] \subseteq \mathbb{R}_{+}$. Assume that the following conditions hold.

(a) $Z: I \mapsto \tilde{\mathcal{A}}$ is an arbitrary process lying in $\operatorname{Ad}(\tilde{\mathcal{A}})_{\text {wac }}$ and there exists positive function $W_{\eta \xi}(t)$ satisfying

$$
\mathbf{d}\left(\frac{d}{d t}<\eta, Z(t) \xi>, P(t, Z(t)(\eta, \xi)) \leq W_{\eta \xi}(t)\right.
$$

(b) Each of the maps $E, F, G, H$ is Lipschitzian from $Q_{Z, \theta}$ to $\left(\operatorname{clos}(\tilde{\mathcal{A}}), \tau_{H}\right)$ where

$$
Q_{Z, \theta}=\left\{(t, x) \in I \times \tilde{\mathcal{A}}:\|x-Z(t)\|_{\eta \xi} \leq \theta, \forall \eta, \xi \in \mathbb{D} \underline{\otimes} \mathbb{E} \text { and }\left\|x_{0}-Z\left(t_{0}\right)\right\|_{\eta \xi} \leq \theta\right\} .
$$

(c) For arbitrary $\eta, \xi \in \mathbb{D} \underline{\otimes} \mathbb{E}, t \in I$,

$$
E_{\eta \xi}(t)=\left\|x_{0}-Z\left(t_{0}\right)\right\|_{\eta \xi} \exp \left(\int_{t_{0}}^{t} d s K_{\eta \xi}^{P}(s)\right)+\int_{t_{0}}^{t} d s W_{\eta \xi}(s) \exp \left(\int_{t_{0}}^{t} d r K_{\eta \xi}^{P}(r)\right) .
$$

If in addition, $E, F, G, H$ are continuous from $I \times \tilde{\mathcal{A}}$ to $\left(\operatorname{clos}(\tilde{\mathcal{A}}), \tau_{H}\right)$, then there exist a solution $\Phi$ of inclusion (1.1) and a subset $J \subseteq I$ such that

$$
\|\Phi(t)-Z(t)\|_{\eta \xi} \leq E_{\eta \xi}(t), \quad t \in J
$$

and

$$
\left|\frac{d}{d t}<\eta, \Phi(t) \xi>-\frac{d}{d t}<\eta, Z(t) \xi>\right| \leq K_{\eta \xi}^{P}(t) E_{\eta \xi}(t)+W_{\eta \xi}(t),
$$

for almost all $t \in J$ where $J=\left\{t \in I: E_{\eta \xi}(t) \leq \theta\right\}$ and $K_{\eta \xi}^{P}: I \mapsto(o, \infty)$ is the Lipschitz function for $P$ lying in $L_{l o c}^{1}(I)$.

Remark 2.2. (i) In this paper, we assume that the coefficients $E, F, G, H$ appearing in (1.1) are Lipschitzian uniformly in $t \in[0,1]$.

Consequently, the map $(t, x) \rightarrow P(t, x)(\eta, x)(\eta, \xi)$ is also Lipschitzian in $x$, uniformly in $t \in[0,1]$. That is for $(t, x),\left(t^{\prime}, y\right) \in[0,1] \times \tilde{\mathcal{A}}$,

$$
\rho\left(P(t, x)(\eta, \xi), P\left(t^{\prime}, y\right)(\eta, \xi)\right) \leq K_{\eta \xi}\|x-y\|_{\eta \xi} .
$$

(ii) By Theorem 2.1, the set of solutions of (1.1) is nonempty and the values of the solutions are contained in the set

$$
Q=A \exp \left(K_{\eta \xi}\right) B \subseteq \theta B
$$

where

$$
B=\left\{x \in \tilde{\mathcal{A}}:\|x\|_{\eta \xi} \leq 1, \forall \eta, \xi \in \mathbb{D} \underline{\otimes} \mathbb{E}\right\}
$$

and $\theta>0$ is some positive fixed number. This can be shown by setting $Z(t) \equiv 0$ and $Z(0)=X^{0}$ in Theorem 2.1. Then by $\mathcal{S}_{(i i i)}$ above,

$$
\mathbf{d}(0, P(t, 0)(\eta, \xi)) \leq A .
$$


Setting $W_{\eta \xi}(t) \equiv A$ in Theorem 2.1, we conclude that there exists a solution $\Phi \in A d(\tilde{\mathcal{A}})_{\text {wac }}$ of (1.1) such that

$$
\|\Phi(t)\|_{\eta \xi} \leq A \exp \left(K_{\eta \xi}\right)
$$

for all $\eta, \xi \in \mathbb{D} \underline{\otimes} \mathbb{E}$. Obviously, $J \times Q \subseteq Q_{0, \theta}$.

Following a similar procedure as in [6], we now introduce the notion of the modulus of continuity for multivalued sesquilinear forms associated with QSDI. Consider a multivalued sesquilinear form

$$
F:[0,1] \rightarrow 2^{\operatorname{Sesq}(\mathbb{I D} \underline{\otimes} \mathbb{E})}
$$

such that $F(t)(\eta, \xi)$ is compact for all $t \in[0,1], \eta, \xi \in \mathbb{D} \underline{\otimes} \mathbb{E}$.

Definition 2.3. (i) The local modulus of continuity for $F(\cdot)(\eta, \xi)$ is defined by

$$
\omega(F ; t, h, \eta, \xi)=\sup \left\{\rho(F(s)(\eta, \xi), F(u)(\eta, \xi)) ; s, u \in\left[t-\frac{h}{2}, t+\frac{h}{2}\right] \bigcap[0,1]\right\} .
$$

(ii) The $L_{p}$ averaged modulus of continuity for $F(\cdot)(\eta, \xi)$ is defined by

$$
\tau(F ; h)(\eta, \xi)_{p}=\left\{\int_{0}^{1}[\omega(F ; t, h, \eta, \xi)]^{p} d t\right\}^{\frac{1}{p}}, 1 \leq p<\infty .
$$

We note that the above modulus is well defined since the map

$$
t \rightarrow \omega(F ; t, h, \eta, \xi)
$$

is measurable and bounded on $[0,1]$. For $p=1$, we simply write

$$
\tau(F ; h)(\eta, \xi)_{1}=\tau(F ; h)(\eta, \xi)
$$

(iii) For $0 \leq t_{1} \leq t_{2} \leq \cdots \leq t_{k} \leq 1$, the $\mathrm{p}$ - variation of the map $t \rightarrow F(t)(\eta, \xi)$ is defined by

$$
W_{p}(F ; \eta, \xi)=\sup \left\{\sum_{i=1}^{k}\left[\rho\left(F\left(t_{i+1}\right)(\eta, \xi), F\left(t_{i}\right)(\eta, \xi)\right)\right]^{p}\right.
$$

Consequent upon the definitions above, we have the following results whose proofs follow similar arguments as in [6].

Theorem 2.4. (i) For $h_{1}<h_{2}$, we have

$$
\tau\left(F ; h_{1}\right)(\eta, \xi)_{p} \leq \tau\left(F ; h_{2}\right)(\eta, \xi)_{p} .
$$


(ii) $\tau\left(F_{1}+F_{2} ; h\right)(\eta, \xi)_{p} \leq \tau\left(F_{1} ; h\right)(\eta, \xi)_{p}+\tau\left(F_{2} ; h\right)(\eta, \xi)_{p}$,

where

$$
\left(F_{1}+F_{2}\right)(t)(\eta, \xi)=F_{1}(t)(\eta, \xi)+F_{2}(t)(\eta, \xi),
$$

the algebraic sum of two mulivalued sesquilinear forms :

$$
F_{1}, F_{2}:[0,1] \rightarrow 2^{\operatorname{Sesq}(\mathbb{D} \underline{\otimes} \mathbb{E})}
$$

(iii) For any positive integer $k$,

$$
\tau(F ; k h)(\eta, \xi) \leq k \tau(F ; h)(\eta, \xi) .
$$

(iv) If $F$ has bounded $\mathrm{p}$ - variation $W_{p}(F ; \eta, \xi)$, then

$$
\tau(F ; h)(\eta, \xi)_{p} \leq\left[W_{p}(F ; \eta, \xi)\right]^{\frac{1}{p}} h^{\frac{1}{p}} .
$$

(v) $\lim _{h \rightarrow 0} \tau(F ; h)(\eta, \xi)=0$ if and only if the map $t \rightarrow F(t)(\eta, \xi)$ is Hausdorff continuous at almost all $t \in[0,1]$.

Next, we fix a partition $0<t_{1}<t_{2} \cdots t_{N}=1$ of the interval $[0,1]$ where $t_{i+1}-t_{i}=h=\frac{1}{N}$ and define the averaged modulus of continuity for the map $(t, x) \rightarrow P(t, x)(\eta, \xi)$. This is needed for the comparison of the set of solutions of (1.1) with the set of solutions of the associated discrete inclusion

$$
\begin{aligned}
<\eta, X_{i+1} \xi> & \in \quad\left\langle\eta, X_{i} \xi>+h P\left(t_{i}, X_{i}\right)(\eta, \xi),\right. \\
X_{0} & =X^{0}, i=0,1 \cdots N-1 .
\end{aligned}
$$

In (2.1), $\left\{X_{i}\right\}_{i=0}^{N}$ is a discrete set of members of $\tilde{\mathcal{A}}$ that approximates $\left\{X\left(t_{i}\right)\right\}, X(t)$ being an exact solution of (1.1). By Theorem 6.3 in [8], inclusion (2.1) is equivalent to the discrete inclusion given by

$$
\begin{array}{r}
X_{i+1} \in X_{i}+\int_{t_{i}}^{t_{i+1}}\left(E\left(t_{i}, X_{i}\right) d \wedge_{\pi}(s)+F\left(t_{i}, X_{i}\right) d A_{f}(s)\right. \\
\left.+G\left(t_{i}, X_{i}\right) d A_{g}^{+}(s)+H\left(t_{i}, X_{i}\right) d s\right),
\end{array}
$$

for approximating QSDI (1.1) in the space $\tilde{\mathcal{A}}$.

Definition 2.5. For fixed $x \in Q, t \in[0,1]$, we adopt the following notations and definitions as in [6]. 
(i) $\omega(P ; t, x, h, \eta, \xi)=\sup \{\rho(P(s, x)(\eta, \xi), P(u, x)(\eta, \xi))$;

$$
\left.s, u \in\left[t-\frac{h}{2}, t+\frac{h}{2}\right] \bigcap[0,1]\right\}
$$

where $\rho(\cdot, \cdot)$ is the Hausdorff metric on $2^{C}$.

(ii) Define the map

$$
\Omega(P ; \cdot, h, \eta, \xi):=\sup \{\omega(P ; \cdot, x, h, \eta, \xi), x \in Q\} .
$$

Then, the map $t \rightarrow \Omega(P ; t, h, \eta, \xi)$ is measurable, bounded and therefore integrable on $[0,1]$.

(iii) The averaged modulus of continuity for the map $P$ is defined by

$$
\tau(P ; h)(\eta, \xi)=\int_{0}^{1} \Omega(P ; t, h, \eta, \xi) d t .
$$

We remark here that Theorem 2.4 (i) - (v) hold for the map $P(t, x)(\eta, \xi)$ appearing in (1.2) provided that in (iv), $P(\cdot, x)(\eta, \xi)$ has bounded $\mathrm{p}$ - variation uniformly in $x \in Q \subseteq \tilde{\mathcal{A}}$, and in (v), $P(\cdot, x)(\eta, \xi)$ is Hausdorff continuous almost everywhere in [0,1] uniformly in $x \in Q$.

\section{ERROR ESTIMATES}

This section is devoted to the establishment of error estimates involved in solving the discretized inclusion (2.1) in place of (1.2). In what follows, let $\eta, \xi \in \mathbb{D} \underline{\otimes} \mathbb{E}$ be arbitrary such that $\eta=c \otimes e(\alpha), \xi=d \otimes e(\beta), c, d \in \mathbb{D}, \alpha, \beta \in L_{\gamma}^{2}\left(\mathbb{R}_{+}\right)$. In solving the discretized inclusion (2.1), one may choose $X_{\eta \xi, i+1}:=<\eta, X_{i+1} \xi>$ arbitrarily from the right hand side of (2.1). However, we employ a definite approach which ensures that these solutions possess certain properties. To this end, we introduce the notion of Lipschitzian quantum stochastic processes.

Definition 3.1. A stochastic process $X:[0,1] \rightarrow \tilde{\mathcal{A}}$ will be said to be Lipschitzian on $[0,1]$ if there exist constants $L_{\eta \xi}>0$ such that

$$
\left\|X\left(t_{1}\right)-X\left(t_{2}\right)\right\|_{\eta \xi} \leq L_{\eta \xi}\left|t_{1}-t_{2}\right|, \forall t_{1}, t_{2} \in[0,1] .
$$

We remark that the set of such processes denoted by $L(\tilde{\mathcal{A}})$, is not empty.

The annihilation process $A:[0,1] \rightarrow \tilde{\mathcal{A}}$ given by

$$
A(t) \xi=\left(\int_{0}^{t} \beta(s) d s\right) \xi, \xi=e(\beta), \beta \in L_{\mathbb{C}, l o c}^{\infty}\left(\mathbb{R}_{+}\right)
$$


satisfies, for $t_{1}, t_{2} \in[0,1]$,

$$
\begin{aligned}
\left\|A\left(t_{1}\right)-A\left(t_{2}\right)\right\|_{\eta \xi} & =\left|<\eta, A\left(t_{1}\right) \xi>-<\eta, A\left(t_{2}\right) \xi>\right| \\
& \leq\left|<\eta, \xi>\| \int_{t_{2}}^{t_{1}} \beta(s) d s\right| \\
& \leq M|<\eta, \xi>|\left|t_{1}-t_{2}\right| \\
& =L_{\eta \xi}\left|t_{1}-t_{2}\right|
\end{aligned}
$$

where

$$
M=\sup _{[0,1]}|\beta(s)|, \quad L_{\eta \xi}=M|<\eta, \xi>| .
$$

Let $\tilde{X} \in L(\tilde{\mathcal{A}}) \cap A d(\tilde{\mathcal{A}})_{\text {wac }}$. We construct the solution $X^{h}$ of the discrete inclusion (2.1) as follows:

$X_{0}^{h}=X^{0}$ and for $i=0,1,2 \cdots N-1$,

$$
\begin{aligned}
X_{\eta \xi, i+1}^{h} & :=<\eta, X_{i+1}^{h} \xi> \\
& =\operatorname{proj}\left(<\eta, \tilde{X}\left(t_{i+1}\right) \xi>,<\eta, X_{i}^{h} \xi>+h P\left(t_{i}, X_{i}^{h}\right)(\eta, \xi)\right),
\end{aligned}
$$

where $\operatorname{proj}(a, A)$ is the unique element of $A$ closest to the element $a \in \mathbb{C}$. The existence and uniqueness of $\operatorname{proj}(a, A)$ are assured by the convexity and compactness of $A \subseteq \mathbb{C}$. Consequently, we have the following results.

Proposition 3.2. Assume that the map $(t, x) \rightarrow P(t, x)(\eta, \xi)$ appearing in (1.2) satisfies the conditions $\mathcal{S}_{(i)}-\mathcal{S}_{(i i i)}$. Then there exists constant $C=C(\eta, \xi)>0$ such that for all $N \geq 2$,

$$
\begin{aligned}
\max _{0 \leq i \leq N}\left\|X_{i}^{h}-\tilde{X}\left(t_{i}\right)\right\|_{\eta \xi} \leq C\left[\left\|\tilde{X}(0)-X^{0}\right\|_{\eta \xi}+\right. \\
\left.\quad+\int_{0}^{1} \mathbf{d}\left(\frac{d}{d t}<\eta, \tilde{X}(t) \xi>, P(t, \tilde{X}(t))(\eta, \xi)\right) d t+\tau(P ; h)(\eta, \xi)+h\right] .
\end{aligned}
$$

Proof. We first show that for any integrable sesquilinear form valued map $f:[a, b] \rightarrow$ $\operatorname{sesq}(\mathbb{D} \underline{\mathbb{Q}} \mathbb{E})$ and any compact and convex set $A \subseteq \mathbb{C}$,

$$
\mathbf{d}\left(\int_{a}^{b} f(t)(\eta, \xi) d t,(b-a) A\right) \leq \int_{a}^{b} \mathbf{d}(f(t)(\eta, \xi), A) d t .
$$

Since

$$
\int_{a}^{b} \operatorname{proj}(A, f(t)(\eta, \xi)) d t \in(b-a) A
$$

then

$$
\begin{aligned}
& \mathbf{d}\left(\int_{a}^{b} f(t)(\eta, \xi) d t,(b-a) A\right) \leq\left|\int_{a}^{b} f(t)(\eta, \xi) d t-\int_{a}^{b} \operatorname{proj}(A, f(t)(\eta, \xi))\right| \\
& \leq \int_{a}^{b}|f(t)(\eta, \xi)-\operatorname{proj}(A, f(t)(\eta, \xi))| d t
\end{aligned}
$$


$=\int_{a}^{b} \mathbf{d}(f(t)(\eta, \xi), A) d t$

Also, for any compact subsets $A, B$ of $\mathscr{C}, y \in \mathbb{C}$, we shall employ the well known inequality:

$$
\mathbf{d}(y, A) \leq \mathbf{d}(y, B)+\rho(A, B) .
$$

Next, for $i=1,2 \cdots N$, we employ the notation

$$
C_{\eta \xi, i}^{h}:=\mathbf{d}\left(<\eta, \tilde{X}\left(t_{i}\right) \xi>,<\eta, \tilde{X}\left(t_{i-1}\right) \xi>+h P\left(t_{i-1}, \tilde{X}\left(t_{i-1}\right)\right)(\eta, \xi)\right) .
$$

Using (3.3) and (3.4), we obtain

$$
\begin{aligned}
& \sum_{i=1}^{N} C_{\eta \xi, i}^{h}=\sum_{i=1}^{N} \mathbf{d}\left(<\eta, \tilde{X}\left(t_{i}\right) \xi>-<\eta, \tilde{X}\left(t_{i-1}\right) \xi>, h P\left(t_{i-1}, \tilde{X}\left(t_{i-1}\right)\right)(\eta, \xi)\right) \\
& \leq \sum_{i=1}^{N} \int_{t_{i}}^{t_{i+1}} \mathbf{d}\left(\frac{d}{d t}<\eta, \tilde{X}(t) \xi>, P\left(t_{i-1}, \tilde{X}\left(t_{i-1}\right)\right)(\eta, \xi)\right) d t \\
& \leq \sum_{i=1}^{N} \int_{t_{i}}^{t_{i+1}}\left[\mathbf{d}\left(\frac{d}{d t}<\eta, \tilde{X}(t) \xi>, P(t, \tilde{X}(t))(\eta, \xi)\right)\right. \\
& \left.+\rho\left(P(t, \tilde{X}(t))(\eta, \xi), P\left(t_{i-1}, \tilde{X}\left(t_{i-1}\right)\right)(\eta, \xi)\right)\right] d t \\
& =\int_{0}^{1} \mathbf{d}\left(\frac{d}{d t}<\eta, \tilde{X}(t) \xi>, P(t, \tilde{X}(t))(\eta, \xi)\right) d t \\
& +\sum_{i=1}^{N} \int_{t_{i}}^{t_{i+1}} \rho\left(P(t, \tilde{X}(t))(\eta, \xi), P\left(t_{i-1}, \tilde{X}\left(t_{i-1}\right)\right)(\eta, \xi)\right) d t .
\end{aligned}
$$

The last term in (3.6) satisfies

$$
\begin{aligned}
& \sum_{i=1}^{N} \int_{t_{i}}^{t_{i+1}} \rho\left(P(t, \tilde{X}(t))(\eta, \xi), P\left(t_{i-1}, \tilde{X}\left(t_{i-1}\right)\right)(\eta, \xi)\right) d t \\
& \leq \sum_{i=1}^{N} \int_{t_{i}}^{t_{i+1}} \rho\left(P(t, \tilde{X}(t))(\eta, \xi), P\left(t_{i}, \tilde{X}\left(t_{i}\right)\right)(\eta, \xi)\right) d t \\
& +\sum_{i=1}^{N} \int_{t_{i}}^{t_{i+1}} \rho\left(P\left(t_{i}, \tilde{X}\left(t_{i}\right)\right)(\eta, \xi), P\left(t_{i-1}, \tilde{X}\left(t_{i-1}\right)\right)(\eta, \xi)\right) d t \\
& \leq \sum_{i=1}^{N} \int_{t_{i}}^{t_{i+1}} \rho\left(P(t, \tilde{X}(t))(\eta, \xi), P\left(t_{i}, \tilde{X}\left(t_{i}\right)\right)(\eta, \xi)\right) d t \\
& +\sum_{i=1}^{N} \int_{t_{i}}^{t_{i+1}} K_{\eta \xi}\left\|\tilde{X}\left(t_{i}\right)-\tilde{X}\left(t_{i-1}\right)\right\|_{\eta \xi} d t \leq \tau(P ; h)(\eta, \xi)+K_{\eta \xi} L_{\eta \xi} h
\end{aligned}
$$

Consequently, from (3.6),

$$
\sum_{i=1}^{N} C_{\eta \xi, i}^{h} \leq \int_{0}^{1} \mathbf{d}\left(\frac{d}{d t}<\eta, \tilde{X}(t) \xi>, P(t, \tilde{X}(t))(\eta, \xi)\right) d t
$$




$$
+K_{\eta \xi} L_{\eta \xi} h+\tau(P ; h)(\eta, \xi)
$$

where $K_{\eta \xi}, L_{\eta \xi}$ are the Lipschitz constants for the map $P(t, \cdot)(\eta, \xi)$ and the process $\tilde{X}$ respectively.

Next, we define $D_{\eta \xi, i}^{h}$ by

$$
D_{\eta \xi, i}^{h}:=\mathbf{d}\left(<\eta, \tilde{X}\left(t_{i}\right) \xi>,<\eta, X_{i-1}^{h} \xi>+h P\left(t_{i-1}, X_{i-1}^{h}\right)(\eta, \xi)\right), i=1,2 \cdots N .
$$

Again, by inequality (3.4),

$D_{\eta \xi, i}^{h} \leq \mathbf{d}\left(<\eta, \tilde{X}\left(t_{i}\right) \xi>,<\eta, \tilde{X}\left(t_{i-1}\right) \xi>+h P\left(t_{i-1}, \tilde{X}\left(t_{i-1}\right)\right)(\eta, \xi)\right)+$

$\rho\left(<\eta, X_{i-1}^{h} \xi>+h P\left(t_{i-1}, X_{i-1}^{h}\right)(\eta, \xi),<\eta, \tilde{X}\left(t_{i-1}\right) \xi>+h P\left(t_{i-1}, \tilde{X}\left(t_{i-1}\right)\right)(\eta, \xi)\right)$

$\leq C_{\eta \xi, i}^{h}+\left\|\tilde{X}\left(t_{i-1}\right)-X_{i-1}^{h}\right\|_{\eta \xi}+h \rho\left(P\left(t_{i-1}, X_{i-1}^{h}\right)(\eta, \xi), P\left(t_{i-1}, \tilde{X}\left(t_{i-1}\right)\right)(\eta, \xi)\right)$

$\leq C_{\eta \xi, i}^{h}+\left(1+h K_{\eta \xi}\right)\left\|\tilde{X}\left(t_{i-1}\right)-X_{i-1}^{h}\right\|_{\eta \xi}$

But by (3.2), the construction of solution $X_{\eta \xi, i}^{h}$ of the discrete inclusion (2.1) satisfies

$$
D_{\eta \xi, i}^{h}=\left|<\eta, \tilde{X}\left(t_{i}\right) \xi>-X_{\eta \xi, i}^{h}\right|=\left\|\tilde{X}\left(t_{i}\right)-X_{i}^{h}\right\|_{\eta \xi} .
$$

Therefore, setting

$$
D_{\eta \xi, 0}^{h}=\left\|\tilde{X}(0)-X^{0}\right\|_{\eta \xi},
$$

we have

$$
D_{\eta \xi, i}^{h} \leq C_{\eta \xi, i}^{h}+\left(1+h K_{\eta \xi}\right) D_{\eta \xi, i-1}^{h}, \quad i=1,2 \cdots N .
$$

By the discrete version of the well known Gronwall inequality (see Dontchev and Farhki [6], for example) we get

$$
\max _{0 \leq i \leq N} D_{\eta \xi, i}^{h} \leq e^{K_{\eta \xi}}\left(\sum_{i=1}^{N} C_{\eta \xi, i}^{h}+D_{\eta \xi, 0}^{h}\right) .
$$

Hence, by (3.7), the conclusion of Proposition (3.2) follows from the last inequality, where the constant $C=\exp \left(K_{\eta \xi}\right)$.

Remark 3.3. If the quantum stochastic process $\tilde{X}$ is a solution of (1.2), then

$$
\left\|\tilde{X}(0)-X^{0}\right\|_{\eta \xi}=0, \mathbf{d}\left(\frac{d}{d t}<\eta, \tilde{X}(t) \xi>, P(t, \tilde{X}(t))(\eta, \xi)\right)=0,
$$

for almost all $t \in[0,1]$. Furthermore, by Theorem 2.3 (iv),

$$
\tau(P ; h)(\eta, \xi) \leq h W(P ; \eta, \xi)
$$


where $W(P ; \eta, \xi)$ is the variation of the map $P$ introduced in Definition 2.2. In particular, if $P(\cdot, x)(\eta, \xi)$ has a bounded variation uniformly in $x \in Q$, then $\tau(P ; h)(\eta, \xi)=O(h)$. Consequently, Proposition 3.2 yields

$$
\max _{0 \leq i \leq N}\left\|\tilde{X}\left(t_{i}\right)-X_{i}^{h}\right\|_{\eta \xi} \leq e^{K_{\eta \xi}}[1+W(P ; \eta, \xi)] h .
$$

The main result of this paper depends partially on the next lemma. To this end, we introduce the following notation.

$$
R_{\eta \xi}:=\sup _{N}\left\{\left|P\left(t_{i}, X_{i}^{N}\right)(\eta, \xi)\right|: X_{\eta \xi}^{N}=\left\{X_{\eta \xi, i}^{N}\right\} \text { solves }(2.1), t_{i} \in[0,1]\right\} .
$$

Lemma 3.4. Let $R_{\eta \xi}$ satisfy (3.8). Then for every solution

$$
X_{\eta \xi}^{N}:=\left\{X_{\eta \xi, i}^{N}=<\eta, X_{i}^{N} \xi>, i=0,1,2 \cdots N\right\}
$$

of $(2.1)$, there exists a solution $\Phi(\cdot)$ of (1.1) such that

$$
\max _{0 \leq i \leq N}\left\|\Phi\left(t_{i}\right)-X_{i}^{N}\right\|_{\eta \xi} \leq \exp \left(K_{\eta \xi}\right)\left(R_{\eta \xi} K_{\eta \xi} h+\tau(P ; h)(\eta, \xi)\right)
$$

Proof. Let a stochastic process $Y^{N}:[0,1] \rightarrow \tilde{\mathcal{A}}$ be defined as follows:

$$
Y^{N}(t)=X_{i}^{N}+\frac{1}{h}\left(t-t_{i}\right)\left(X_{i+1}^{N}-X_{i}^{N}\right), t_{i} \leq t \leq t_{i+1}, i=0,1,2 \cdots N-1 .
$$

The associated piecewise linear matrix element $Y_{\eta \xi}^{N}(t):=<\eta, Y^{N}(t) \xi>$ is given by

$$
Y_{\eta \xi}^{N}(t)=X_{\eta \xi, i}^{N}+\frac{1}{h}\left(t-t_{i}\right)\left(X_{\eta \xi, i+1}^{N}-X_{\eta \xi, i}^{N}\right)
$$

where $\left\{X_{\eta \xi, i}^{N}\right\}$ is a set of grid solutions of (2.1). Clearly, $Y^{N} \in \operatorname{Ad}(\tilde{\mathcal{A}})_{w a c}$.

By construction we have

$$
\frac{d}{d t} Y_{\eta \xi}^{N}(t)=\frac{1}{h}\left(X_{\eta \xi, i+1}^{N}-X_{\eta \xi, i}^{N}\right) \in P\left(t_{i}, X_{i}^{N}\right)(\eta, \xi) .
$$

Next, we estimate

$$
\mathbf{d}\left(\frac{d}{d t} Y_{\eta \xi}^{N}(t), P\left(t, Y^{N}(t)\right)(\eta, \xi)\right)
$$

By inequality (3.4),

$$
\begin{aligned}
& \mathbf{d}\left(\frac{d}{d t} Y_{\eta \xi}^{N}(t), P\left(t, Y^{N}(t)\right)(\eta, \xi)\right) \\
& \leq \mathbf{d}\left(\frac{d}{d t} Y_{\eta \xi}^{N}(t), P\left(t, X_{i}^{N}\right)(\eta, \xi)\right)+\rho\left(P\left(t, Y^{N}(t)\right)(\eta, \xi), P\left(t, X_{i}^{N}\right)(\eta, \xi)\right)
\end{aligned}
$$


$\leq \mathbf{d}\left(\frac{d}{d t} Y_{\eta \xi}^{N}(t), P\left(t, X_{i}^{N}\right)(\eta, \xi)\right)+K_{\eta \xi}\left|Y_{\eta \xi}^{N}(t)-X_{\eta \xi, i}^{N}\right|$

$\leq \mathbf{d}\left(\frac{d}{d t} Y_{\eta \xi}^{N}(t), P\left(t, X_{i}^{N}\right)(\eta, \xi)\right)+K_{\eta \xi} R_{\eta \xi} h$

The last inequality follows from (3.8) and (3.10). Again, by employing (3.11) and (3.4), we have

$$
\begin{aligned}
& \mathbf{d}\left(\frac{d}{d t} Y_{\eta \xi}^{N}(t), P\left(t, X_{i}^{N}\right)(\eta, \xi)\right) \\
\leq & \rho\left(P\left(t_{i}, X_{i}^{N}\right)(\eta, \xi), P\left(t, X_{i}^{N}\right)(\eta, \xi)\right) \\
\leq & \omega\left(P ; X_{i}^{N}, t, 2 h, \eta, \xi\right) \\
\leq & \sup _{x \in Q} \omega(P ; x, t, 2 h, \eta, \xi)=\Omega(P ; t, 2 h, \eta, \xi) .
\end{aligned}
$$

Applying Theorem 2.1 by setting

$$
W_{\eta \xi}(t)=K_{\eta \xi} R_{\eta \xi} h+\Omega(P ; t, 2 h, \eta, \xi),
$$

we conclude that there exists a solution $\Phi(\cdot)$ of (1.1) satisfying inequality (3.9).

Next, we present our main result. To this end, we introduce the following sets of vectors in the space $\mathbb{C}^{N+1}$.

Denote by $S\left(X^{0}\right)$ the subset of $A d(\tilde{\mathcal{A}})_{w a c}$ consisting of the trajectory bundle of (1.1). Then, we define the following sets.

$$
\begin{aligned}
& R^{N}\left(X^{0}\right)(\eta, \xi):=\left\{\Phi_{\eta \xi}^{N}=\left(\Phi_{\eta \xi}\left(t_{i}\right), i=0,1,2 \cdots N\right),\right. \\
& \text { such that } \left.\Phi(\cdot) \in S\left(X^{0}\right)\right\} . \\
& D^{N}\left(X^{0}\right)(\eta, \xi):=\left\{X_{\eta \xi}^{N}=\left(X_{\eta \xi, i}, i=0,1,2 \cdots N\right),\right.
\end{aligned}
$$

such that $X_{\eta \xi}^{N}$ is a solution of the discretized inclusion (2.1)\}

For $U, V \in \mathbb{C}^{N+1}$, we employ the maximum norm

$$
|U-V|:=\max _{0 \leq i \leq N}\left|U_{i}-V_{i}\right|
$$

and the associated Hausdorff metric $\rho$ on $2^{C^{N+1}}$.

Consequently, the following theorem has been established by the combination of Proposition 3.2 and Lemma 3.4 .

Theorem 3.5. Assume that the QSDI (1.2) satisfies conditions $\mathcal{S}_{(i)}-\mathcal{S}_{(i i i)}$. Then there exist positive constants depending only on $\eta, \xi$ such that

$$
\rho\left(R^{N}\left(X^{0}\right)(\eta, \xi), D^{N}\left(X^{0}\right)(\eta, \xi)\right) \leq C[\tau(P ; h)(\eta, \xi)+h],
$$


for all $N \geq 2$.

\section{ACKNOWLEDGEMENT}

I am grateful to the Abdus Salam International Centre for Theoretical Physics, Trieste, Italy, for a visiting Research Fellowship in Mathematics through which this work was done. I thank Professor M. Virasoro, the Director of the Centre and Professor A. O. Kuku of Mathematics Section, for his help and encouragement. 


\section{REFERENCES}

[1] Aubin, J.P; Cellina, A. : Differential Inclusions, Springer - Verlag, (1984).

[2] Aubin, J.P; Frankowska, H. : Set - Valued Analysis, Birkhauser, (1990).

[3] Ayoola, E.O. : Exponential formula for the reachable sets of quantum stochastic differential inclusions, submitted to Stochastic Analysis and Applications.

[4] Ayoola, E.O.: Construction of approximate attainability sets for Lipschitzian quantum stochastic differential inclusions, Stochastic Analysis and Applications 19(3), (2001) 461471.

[5] Ayoola, E.O.: On convergence of one - step schemes for weak solutions of quantum stochastic differential equations, Acta Applicandae Mathematicae, 67(1), (May, 2001) 19 - 58.

[6] Dontchev, A.L; Farkhi, E.M : Error estimates for discretized differential inclusions, Computing 41 (1989) 349-358.

[7] Dontchev, A; Lempio, F.: Difference methods for differential inclusions: A survey, SIAM Rev. 34(2), (1992) 263-294.

[8] Ekhaguere, G.O.S. : Lipschitzian quantum stochastic differential inclusions, Internat. J. Theoret. Phys. 31(11), (1992) 2003-2034.

[9] Ekhaguere, G.O.S.: Quantum stochastic differential inclusions of hypermaximal monotone type, Internat. J. Theoret. Phys. 34(3), (1995), 323-353.

[10] Ekhaguere, G.O.S.: Quantum stochastic evolutions. Internat. J. Theoret. Phys. 35(9), (1996), $1909-1946$.

[11] Hudson, R.L; Parthasarathy, K.R.: Quantum Ito's formulae and stochastic evolutions, Commun. Math. Phys. 93, (1984) 301-324

[12] V.A.Komarov, V.A; Pevchikh, K.E. : A method of approximating attainability sets for differential inclusions with a specified accuracy, Comput. Maths. Math. Phys, 31(1) (1991) 109-112.

[13] Veliov, V.: Second order discrete approximation to linear differential inclusions, SIAM J. Num. Anal. 29, (1992) 439-451.

[14] Wolenski, Peter R. : A uniqueness theorem for differential inclusions, J.Differential Eq.,84 (1990), 165-182.

[15] Wolenski, Peter R. : The exponential formula for the reachable set of a Lipschitz differential inclusion, SIAM J. Control Optim. 28(5), (1990) 1148-1161. 